\title{
Research Article \\ Output Regulation Problem for Differentiable Families of Linear Systems
}

\author{
Albert Compta, Josep Ferrer, and Marta Peña \\ Departament de Matemàtica Aplicada I, Escola Tècnica Superior d'Enginyeria Industrial de Barcelona, \\ Universitat Politècnica de Catalunya, Diagonal 647, 08028 Barcelona, Spain \\ Correspondence should be addressed to Marta Peña, marta.penya@upc.edu \\ Received 9 April 2010; Revised 12 July 2010; Accepted 12 July 2010 \\ Academic Editor: Oded Gottlieb \\ Copyright (C) 2010 Albert Compta et al. This is an open access article distributed under the Creative \\ Commons Attribution License, which permits unrestricted use, distribution, and reproduction in \\ any medium, provided the original work is properly cited. \\ Given a family of linear systems depending on a parameter varying in a differentiable manifold, we \\ obtain sufficient conditions for the existence of a (global or local) differentiable family of controllers \\ solving the output regulation problem for the given family. Moreover, we construct it when these \\ conditions hold.
}

\section{Introduction}

The output regulation problem arose as one of the main research topics in linear control theory in the 1970s. This problem considers controlling a given plant such that its output tracks a reference signal or rejects a disturbance. The reference and disturbance signals are typically generated by an external system called exosystem. The output regulation problem for linear time-invariant systems has been well studied by many authors like, for example, [1-8]. Usually, these LTI systems are obtained by linearizing around an operating point or by using system identification techniques. However, in some cases this approach is restrictive, and it would be better to allow the system to depend differentiably on a specific parameter. We think that this situation is interesting to consider and, in this case, the controller must be able to maintain the properties of closed-loop stability and output regulation when it is modelled as a global or a local differentiable family. We notice that recently a regulator design approach based on the parameterization of a set of controllers that can achieve regulation for switched bimodal linear systems has been presented in [9].

Thus, we lead to the quite general question of whether pointwise solvability implies the existence of a nicely parameterized solution [10]. There is an abundance of literature concerning global parameterized families of linear systems, and in particular the key point of global pole assignment, mainly when pointwise controllability holds. 
For a general introduction to families of linear systems, see, for example, [11]. Some problems that justify the study of families of systems are presented there, and one tackles the classification of families (fine moduli spaces), the existence of global canonical forms, and some others. In fact, [11] deals with a more general class of families of linear systems in terms of bundles over the space of parameters, which includes the parameterized ones. An alternative generalization is the consideration of systems over rings, that is to say, pairs of matrices with entries on a commutative ring. The particular case of parameterized families arise, when rings of functions defined in the space of parameters are considered. See [12] for a general introduction and [10] for a survey and many references mainly centered on control and stabilization problems.

In particular, the global pole assignment of parameterized families has been widely studied (see, e.g., [10]), always under the hypothesis of pointwise controllability. The problem is solved in $[13,14]$ by means of algebrogeometric and algebraic techniques, respectively, if provided constant controllability indices. Other conditions are considered, for example, in [15] (constant rank of $B$, ring controllability) or in [16] (one-dimensional manifold of parameters).

The general case of nonnecessarily pointwise controllable families is solved in [17]. In fact, it provides a general machinery for extending the local case to the global parametrical one if one has a geometrical description of the construction in the constant case. Notice that this method does not need further references to algebrogeometric or algebraic techniques, so that it seems more accessible to engineers. Here, we show that this machinery is also applicable to more sophisticated problems, such as the construction of global families of suitable controllers.

Secondly, we tackle the same problem for local differentiable perturbations of the systems, that is to say, for parameterized families defined not globally on a (contractible) manifold but locally in some neighborhood of the original point. The pointwise construction is easily extended for the controllable case, but up to our knowledge no technique exists for the general case (beyond the trivial approach as the restriction of a global family). In this sense, a significative contribution is [18], where one proves that local differentiable pole assignment exists (even for the noncontrollable case) without any pointwise hypothesis on the local family of perturbations, in contrast with the global case above. The proof is based in the use of Arnold's techniques, reducing the problem to a suitable versal deformation of the central system. Here we show that this quite surprising result can be used in more complex processes, such as the output regulation.

In summary, this work deals with the existence of differentiable families of controllers following the pattern in [4], both for global and local families of systems. In particular, we assume that the hypotheses there hold. We will denote them by (F1)-(F7) (see Theorem 2.1), and we add (') for the natural generalization to parameterized families (see Theorems 3.3 and 4.2). We remark (see [4]) that (F1)-(F6) either involve no loss of generality or are necessary for the existence of the controller.

As we have pointed out, the assumptions concerning controllability or detectability of a system are easily transferred from the pointwise case to parameterized families. Obstructions arise when subsystems are considered and in general for geometrical conditions. The key tools are the techniques in [17] for the global case and the results in [18] for the local one.

The paper is organized as follows. First we present, as a reminder, the main result in [4], emphasizing the pointwise hypotheses that will be considered later. In the following sections we deal with the global and the local cases. 
Notations $\mathbb{R}$ and $\mathbb{C}$ denote the fields of real and complex numbers, respectively. $\overline{\mathbb{C}^{+}}$is the closed right-half complex plane and $\mathbb{C}^{-}$is the open left-half one. If $v_{1}, \ldots, v_{s}$ are vectors of $\mathbb{R}^{n}$, then $\left[v_{1}, \ldots, v_{s}\right]$ denotes the subspace spanned by them. We write $M_{n, m}(\mathbb{R})$ for the vector space of matrices with $n$ rows and $m$ columns with entries in $\mathbb{R}$. We identify $M_{n, \mathrm{n}+m}(\mathbb{R})$ with the pairs of matrices $M_{n, n}(\mathbb{R}) \times M_{n, m}(\mathbb{R})$. If $A$ is a matrix, $A^{t}$ is its transpose matrix. $I$ denotes the identity matrix and $I_{k}$ the identity $k$-matrix. $\sigma(\cdot)$ denotes the spectrum of the corresponding matrix or pairs of matrices, each eigenvalue being repeated as many times as its multiplicity. For linear spaces $\mathcal{R}$ and $\mathcal{S}, \mathcal{R} \cong \mathcal{S}$ means that $R$ and $\mathcal{S}$ are isomorphic and $\operatorname{Hom}(\mathcal{R}, \mathcal{S})$ is the linear space of all linear maps $\mathcal{R} \rightarrow \mathcal{S}$.

\section{Preliminaries: The Nonparameterized Case}

In this section, we summarize the problem and the results stated in [4], which have been the basis of our work. This reference deals with the regulation of the linear system represented by

$$
\begin{gathered}
\dot{x}_{1}=A_{1} x_{1}+A_{3} x_{2}+B_{1} u, \\
\dot{x}_{2}=A_{2} x_{2}, \\
y=C_{1} x_{1}+C_{2} x_{2}, \\
z=D_{1} x_{1}+D_{2} x_{2},
\end{gathered}
$$

where $x_{1}$ is the plant state vector, $u$ the control input, $x_{2}$ the vector of exogenous signals, $y$ the vector of measurements available for control, and $z$ the output to be regulated. These vectors belong to fixed finite-dimensional real linear spaces $x_{1}, \mathfrak{U}, \boldsymbol{x}_{2}, \mathcal{y}$, and $\mathfrak{z}$, of dimensions $n_{1}$, $m, n_{2}, n_{y}$, and $n_{z}$, respectively; the linear maps or real matrices $A_{1}, A_{2}, A_{3}, B_{1}, C_{1}, C_{2}, D_{1}$, and $\mathrm{D}_{2}$ are time-invariant.

The objective is to construct a controller modelled by the equations

$$
\begin{aligned}
& \dot{x}_{c}=A_{c} x_{c}+B_{c} y, \\
& u=F_{c} x_{c}+G_{c} y,
\end{aligned}
$$

where $x_{c}$ is the compensator state vector, which belongs to a finite-dimensional real linear space $\mathcal{X}_{c}$, and the linear maps $A_{c}, B_{c}, F_{c}$, and $G_{c}$ are time-invariant. This system must achieve two properties: closed-loop stability and output regulation of the associated closed loop defined as

$$
\begin{gathered}
\dot{x}_{L}=A_{L} x_{L}+B_{L} x_{2}, \\
z=D_{L} x_{L}+D_{2} x_{2},
\end{gathered}
$$


where

$$
\begin{gathered}
x_{L}=\left(\begin{array}{l}
x_{1} \\
x_{c}
\end{array}\right), \quad x_{L}=x_{1} \oplus x_{c} \\
A_{L}=\left(\begin{array}{cc}
A_{1}+B_{1} G_{c} C_{1} & B_{1} F_{c} \\
B_{c} C_{1} & A_{c}
\end{array}\right), \quad B_{L}=\left(\begin{array}{c}
A_{3}+B_{1} G_{c} C_{2} \\
B_{c} C_{2}
\end{array}\right), \quad D_{L}=\left(\begin{array}{ll}
D_{1} & 0
\end{array}\right) .
\end{gathered}
$$

Closed-loop stability means that $A_{L}$ is stable, that is, $\sigma\left(A_{L}\right) \subset \mathbb{C}^{-}$, and output regulation means that $z(t) \rightarrow 0$ as $t \rightarrow \infty$ for all $x_{L}(0)$ and $x_{2}(0)$.

We next introduce the mathematical setting used in [4]. For any linear space $R$, bring in the linear space $\underline{R}=\operatorname{Hom}\left(\mathcal{X}_{2}, \mathcal{R}\right)$. Given maps $A: \mathcal{X} \rightarrow \mathcal{X}$ and $C: \mathcal{X} \rightarrow \mathcal{y}$, define, respectively, the linear maps $\underline{A}: \underline{x} \rightarrow \underline{x}$ and $\underline{C}: \underline{x} \rightarrow \underline{y}$ by

$$
\underline{A X}=A X-X A_{2}, \quad \underline{C} X=C X, \quad X \in X .
$$

Analogous notation will be used for the subsystems involved in the construction.

Now we state the main result obtained in [4].

Theorem 2.1 (see [4]). Assume that

(F1) $\sigma\left(A_{2}\right) \subset \overline{\mathbb{C}^{+}}$,

(F2) $\operatorname{Im} C_{1}+\operatorname{Im} C_{2}=y$,

(F3) $\operatorname{Im} D_{1}=\mathfrak{z}$,

(F4) $\left(A_{1}, B_{1}\right)$ is stabilizable,

(F5) $\left(C_{1}, A_{1}\right)$ is detectable,

(F6) $(C, A)$ is detectable, where $A=\left(\begin{array}{cc}A_{1} & A_{3} \\ 0 & A_{2}\end{array}\right)$ and $C=\left(\begin{array}{ll}C_{1} & C_{2}\end{array}\right)$.

Then, a controller exists if and only if

(F7) $\left(\begin{array}{l}A_{3} \\ D_{2}\end{array}\right) \in \operatorname{Im}\left(\begin{array}{ll}\underline{A}_{1} & \underline{B}_{1} \\ \underline{D}_{1} & 0\end{array}\right)$.

Following [4] again, an algorithm to compute a controller can be organized into the following four steps.

Step 1. Select $B_{c}$ so that $A-B_{c} C$ is stable.

Step 2. Select $F_{1}$ so that $A_{1}+B_{1} F_{1}$ is stable.

Step 3. Select $F_{2}$ so that

$$
\left(\begin{array}{c}
A_{3}+B_{1} F_{2} \\
D_{2}
\end{array}\right) \in \operatorname{Im}\left(\begin{array}{c}
\underline{A}_{1}+\underline{B}_{1} \underline{F}_{1} \\
\underline{D}_{1}
\end{array}\right)
$$

Step 4. Set $F_{c}=\left(\begin{array}{ll}F_{1} & F_{2}\end{array}\right), A_{c}=A-B_{c} C+B F_{c}$, and $G_{c}=0$. 


\section{The Globally Parameterized Case}

Our aim is to generalize the above results when the involved matrices depend on a parameter varying in a differentiable manifold. That is to say, we consider a family of linear systems depending differentiably on a parameter, and we study sufficient conditions to ensure the existence of a differentiable solution of the output regulation problem for the given family.

Thus, we consider a differentiable family of linear systems modelled by the equations

$$
\begin{gathered}
\dot{x}_{1}=A_{1}(\tau) x_{1}+A_{3}(\tau) x_{2}+B_{1}(\tau) u \\
\dot{x}_{2}=A_{2}(\tau) x_{2} \\
y=C_{1}(\tau) x_{1}+C_{2}(\tau) x_{2} \\
z=D_{1}(\tau) x_{1}+D_{2}(\tau) x_{2}
\end{gathered}
$$

or, equivalently, it will be represented by a differentiable family of matrices $\left(A_{1}(\tau), A_{2}(\tau), A_{3}(\tau), B_{1}(\tau), C_{1}(\tau), C_{2}(\tau), D_{1}(\tau), D_{2}(\tau)\right)$.

To this end, basic tools are introduced in [19]. More specifically, we will need the following result.

Lemma 3.1. Let $M$ be a contractible manifold. If $A(\tau), \tau \in M$, is a differentiable family of $q \times p$ real matrices having constant rank and $v(\tau), \tau \in M$, a differentiable family of vectors in $\operatorname{Im} A(\tau) \subset \mathbb{R}^{q}$, then there exists a differentiable family of vectors $u(\tau) \in \mathbb{R}^{p}$ such that

$$
A(\tau) u(\tau)=v(\tau)
$$

for any $\tau \in M$.

Proof. In [19, (IV-1-6)], it is proved that $\operatorname{Im} A(\tau)$ and $\operatorname{Ker} A(\tau)$ are differentiable families of subspaces of $\mathbb{R}^{q}$ and $\mathbb{R}^{p}$, respectively. Then, from [19, (IV-2-3)], we can choose differentiable families $u_{1}(\tau), \ldots, u_{s}(\tau)$ of linearly independent vectors such that

$$
\mathbb{R}^{p}=\operatorname{Ker} A(\tau) \oplus\left[u_{1}(\tau), \ldots, u_{s}(\tau)\right]
$$

for every $\tau \in M$. Clearly $A(\tau) u_{1}(\tau), \ldots, A(\tau) u_{s}(\tau)$ is a differentiable basis of $\operatorname{Im} A(\tau)$. So, the coordinates of $v(\tau)$ in this basis vary differentiably, that is, there exist differentiable families $a_{1}(\tau), \ldots, a_{s}(\tau)$ of real numbers such that

$$
v(\tau)=a_{1}(\tau) A(\tau) u_{1}(\tau)+\cdots+a_{s}(\tau) A(\tau) u_{s}(\tau)
$$

Finally, it suffices to take

$$
u(\tau)=a_{1}(\tau) u_{1}(\tau)+\cdots+a_{S}(\tau) u_{s}(\tau) .
$$


In addition, we will use the following result from [17], where these tools are applied to prove the existence of a global parameterized pole assignment. Recall that if $M$ is a contractible manifold and $(A(\tau), B(\tau)), \tau \in M$, is a differentiable family of pairs of matrices of $M_{n, n+m}(\mathbb{R})$, the family $(A(\tau), B(\tau))$ has constant Brunovsky type if

(i) the controllability indices are constant,

(ii) the Jordan invariants have constant type, that is to say, the number of distinct eigenvalues and the list of sizes of the Jordan blocks corresponding to different eigenvalues are independent of $\tau$.

Theorem 3.2 (see [17]). Let $M$ be a contractible manifold, $(A(\tau), B(\tau)), \tau \in M$, a differentiable family of pairs of matrices of $M_{n, n+m}(\mathbb{R})$ having constant Brunovsky type, $\lambda_{1}(\tau), \ldots, \lambda_{q}(\tau) \in$ $\mathbb{C}$ giving the distinct eigenvalues of $(A(\tau), B(\tau))$, and $m_{1}, \ldots, m_{q}$ their respective algebraic multiplicities. If $\mu_{i}(\tau) \in \mathbb{C}, 1 \leq i \leq s$, is a set of maps closed under conjugation, then there exists a differentiable family of matrices $F(\tau) \in M_{m, n}(\mathbb{R})$ such that the eigenvalues of $A(\tau)+B(\tau) F(\tau)$ are $\mu_{1}(\tau), \ldots, \mu_{s}(\tau), \lambda_{1}(\tau), \ldots, \lambda_{q}(\tau)$, with the latter having multiplicities $m_{1}, \ldots, m_{q}$.

These results allow us to study the existence of a global differentiable family of controllers. In the next section we address the local case.

Theorem 3.3. Let $M$ be a contractible manifold and $\left(A_{1}(\tau), A_{2}(\tau), A_{3}(\tau), B_{1}(\tau), C_{1}(\tau), C_{2}(\tau)\right.$, $\left.D_{1}(\tau), D_{2}(\tau)\right), \tau \in M$, a differentiable family of linear systems verifying that for any $\tau \in M$

$\left(\mathrm{F} 1^{\prime}\right) \sigma\left(A_{2}(\tau)\right) \subset \overline{\mathbb{C}^{+}}$,

(F2') $\operatorname{Im} C_{1}(\tau)+\operatorname{Im} C_{2}(\tau)=y$,

(F3') $\operatorname{Im} D_{1}(\tau)=$ Z,

(F4') $\left(A_{1}(\tau), B_{1}(\tau)\right)$ is stabilizable,

(F5') $\left(C_{1}(\tau), A_{1}(\tau)\right)$ is detectable,

$\left(\mathrm{F}^{\prime}\right)(C(\tau), A(\tau))$ is detectable,

$\left(\mathrm{F}^{\prime}\right)\left(\begin{array}{l}A_{3}(\tau) \\ D_{2}(\tau)\end{array}\right) \in \operatorname{Im}\left(\begin{array}{cc}\underline{A}_{1}(\tau) & \underline{B}_{1}(\tau) \\ \underline{1}_{1}(\tau) & 0\end{array}\right)$.

Then, there exists a global differentiable family of controllers $\left(A_{c}(\tau), B_{c}(\tau), F_{c}(\tau), G_{c}(\tau)\right)$ if, when $\tau$ varies in $M$, one has:

(i) $(C(\tau), A(\tau))$ has constant Brunovsky type,

(ii) $\left(A_{1}(\tau), B_{1}(\tau)\right)$ has constant Brunovsky type,

(iii) $\operatorname{rank}\left(\begin{array}{cc}I_{n_{2}} \otimes A_{1}(\tau)-A_{2}(\tau)^{t} \otimes I_{n_{1}} & I_{n_{2}} \otimes B_{1}(\tau) \\ I_{n_{2}} \otimes D_{1}(\tau) & 0\end{array}\right)$ is constant.

Proof. Let us see that conditions (i), (ii), and (iii) allow us to transfer the pointwise algorithm in [4] summarized in the above section to the parameterized case.

Step 1. If condition (i) holds, we can select a differentiable family $B_{c}(\tau)$ such that $A(\tau)-$ $B_{c}(\tau) C(\tau)$ is stable, for any $\tau \in M$, by means of Theorem 3.2: $\lambda_{1}(\tau), \ldots, \lambda_{q}(\tau) \in \mathbb{C}^{-}$because of $\left(\mathrm{F}^{\prime}\right)$, so that it suffices to take $\mu_{1}(\tau), \ldots, \mu_{s}(\tau) \in \mathbb{C}^{-}$.

Step 2. Analogously, if condition (ii) holds, we can select a differentiable family $F_{1}(\tau)$ such that $A_{1}(\tau)+B_{1}(\tau) F_{1}(\tau)$ is stable, for any $\tau \in M$. 
Step 3. By hypothesis (F7'),

$$
\left(\begin{array}{l}
A_{3}(\tau) \\
D_{2}(\tau)
\end{array}\right) \in \operatorname{Im}\left(\begin{array}{cc}
\underline{A}_{1}(\tau) & \underline{B}_{1}(\tau) \\
\underline{D}_{1}(\tau) & 0
\end{array}\right)
$$

and with $\operatorname{rank}\left(\begin{array}{cc}\underline{A}_{1}(\tau) & \underline{B}_{1}(\tau) \\ \underline{D}_{1}(\tau) & 0\end{array}\right)$ being constant (because of condition (iii)) it is guaranteed that the dimension of this subspace is constant in $M$. Using the Kronecker product and the vecfunction, this condition can be reformulated as condition (iii) in terms of the matrices defining the system.

(F7') turns to

Right multiplying by matrix $\left(\begin{array}{cc}I & 0 \\ \underline{F}_{1}(\tau) & I\end{array}\right)$, this dimension does not change and hypothesis

$$
\left(\begin{array}{l}
A_{3}(\tau) \\
D_{2}(\tau)
\end{array}\right) \in \operatorname{Im}\left(\begin{array}{cc}
\underline{A}_{1}(\tau)+\underline{B}_{1}(\tau) \underline{F}_{1}(\tau) & \underline{B}_{1}(\tau) \\
\underline{D}_{1}(\tau) & 0
\end{array}\right) .
$$

Then, from Lemma 3.1 there exist differentiable families $X(\tau), Y(\tau)$ such that

$$
\left(\begin{array}{c}
A_{3}(\tau) \\
D_{2}(\tau)
\end{array}\right)=\left(\begin{array}{cc}
\underline{A}_{1}(\tau)+\underline{B}_{1}(\tau) \underline{F}_{1}(\tau) & \underline{B}_{1}(\tau) \\
\underline{D}_{1}(\tau) & 0
\end{array}\right)\left(\begin{array}{l}
X(\tau) \\
Y(\tau)
\end{array}\right)
$$

or, equivalently,

$$
\begin{gathered}
A_{3}(\tau)-\underline{B}_{1}(\tau) Y(\tau)=\left(\underline{A}_{1}(\tau)+\underline{B}_{1}(\tau) \underline{F}_{1}(\tau)\right) X(\tau), \\
D_{2}(\tau)=\underline{D}_{1}(\tau) X(\tau) .
\end{gathered}
$$

Therefore, taking $F_{2}(\tau)=-Y(\tau)$, we have

$$
\left(\begin{array}{c}
A_{3}(\tau)+B_{1}(\tau) F_{2}(\tau) \\
D_{2}(\tau)
\end{array}\right) \in \operatorname{Im}\left(\begin{array}{c}
\underline{A}_{1}(\tau)+\underline{B}_{1}(\tau) \underline{F}_{1}(\tau) \\
\underline{D}_{1}(\tau)
\end{array}\right)
$$

for any $\tau \in M$.

Step 4. Set $F_{c}(\tau)=\left(F_{1}(\tau) \quad F_{2}(\tau)\right), A_{c}(\tau)=A(\tau)-B_{c}(\tau) C(\tau)+B(\tau) F_{c}(\tau), G_{c}(\tau)=0$.

Clearly, they are differentiable families in $M$, and, from Theorem 2.1, they define a controller for any $\tau \in M$.

\section{The Locally Parameterized Case}

In this section, we tackle the local case, that is, we deal with local differentiable families. This means that the parameter varies only in an open neighborhood of the origin of $\mathbb{R}^{k}$.

Similarly to the global case, we will use the existence of a local differentiable family of pole assignments for a local differentiable family of stabilizable pairs. As above, a pointwise 
construction does not guarantee the differentiability of the family of feedbacks. It is so by means of the following result, based on the Arnold's techniques about versal deformations.

Theorem 4.1 (see [18]). Let $(A, B) \in M_{n, n+m}(\mathbb{R})$ be a stabilizable pair and $(A(\tau), B(\tau)), \tau \in V$, a local differentiable family of pairs with $(A(0), B(0))=(A, B)$. Then there is a local differentiable family of feedbacks $F(\tau) \in M_{m, n}(\mathbb{R})$, defined in some open neighborhood of the origin $W \subset V$, such that $\sigma(A(\tau)+B(\tau) F(\tau)) \subset \mathbb{C}^{-}$.

Finally, we apply this result to our output regulation problem.

Theorem 4.2. Let $\left(A_{1}(\tau), A_{2}(\tau), A_{3}(\tau), B_{1}(\tau), C_{1}(\tau), C_{2}(\tau), D_{1}(\tau), D_{2}(\tau)\right)$ be a differentiable family of linear systems defined in some open neighborhood of the origin $V \subset \mathbb{R}^{k}$. Assume that, for any $\tau \in V$,

$\left(\mathrm{F} 1^{\prime}\right) \sigma\left(A_{2}(\tau)\right) \subset \overline{\mathbb{C}^{+}}$,

(F2) $\operatorname{Im} C_{1}+\operatorname{Im} C_{2}=y$,

(F3) $\operatorname{Im} D_{1}=\mathfrak{Z}$

(F4) $\left(A_{1}, B_{1}\right)$ is stabilizable,

(F5) $\left(C_{1}, A_{1}\right)$ is detectable,

(F6) $(C, A)$ is detectable,

$\left(\mathrm{F}^{\prime}\right)\left(\begin{array}{c}A_{3}(\tau) \\ D_{2}(\tau)\end{array}\right) \in \operatorname{Im}\left(\begin{array}{ll}\underline{A}_{1}(\tau) & \underline{B}_{1}(\tau) \\ \underline{D}_{1}(\tau) & 0\end{array}\right)$.

Then, there exists a local differentiable family of controllers $\left(A_{c}(\tau), B_{c}(\tau), F_{c}(\tau), G_{c}(\tau)\right)$ in some open neighborhood of the origin $W \subset V$ if, when $\tau$ varies in $V$, one has:

(iii) $\operatorname{rank}\left(\begin{array}{cc}I_{n_{2}} \otimes A_{1}(\tau)-A_{2}(\tau)^{t} \otimes I_{n_{1}} & I_{n_{2}} \otimes B_{1}(\tau) \\ I_{n_{2}} \otimes D_{1}(\tau) & 0\end{array}\right)$ is constant.

Proof. It is easy to adapt the proof of Theorem 3.3 bearing in mind the following remarks.

Notice that hypotheses (F2) and (F3) mean, respectively, that matrices $\left(\begin{array}{lll}C_{1} & C_{2}\end{array}\right)$ and $D_{1}$ have maximal rank. Hence, matrices $\left(C_{1}(\tau) \quad C_{2}(\tau)\right)$ and $D_{1}(\tau)$ have maximal rank for any $\tau$ small enough or, equivalently,

$$
\operatorname{Im} C_{1}(\tau)+\operatorname{Im} C_{2}(\tau)=y, \quad \operatorname{Im} D_{1}(\tau)=\mathfrak{z} .
$$

In a similar way, since the set of detectable pairs is open, hypothesis (F5) guarantees that pairs $\left(C_{1}(\tau), A_{1}(\tau)\right)$ are detectable for $\tau$ small enough.

Moreover, hypotheses (F4) and (F6) allow us to apply Theorem 4.1 in the first two steps of the synthesis algorithm. Notice that, thanks to Theorem 4.1, hypotheses (i) and (ii) can be avoided. Finally, for Step 3 we use (F7') and (iii) as in the proof of Theorem 3.3.

\section{Acknowledgment}

This work was supported by the Ministerio de Educación y Ciencia under Project MTM200767812-C02-02. 


\section{References}

[1] E. J. Davison and A. Goldenberg, "Robust control of a general servomechanism problem: the servo compensator," Automatica, vol. 11, no. 5, pp. 461-471, 1975.

[2] E. J. Davison, "The robust control of a servomechanism problem for linear time-invariant multivariable systems," IEEE Transactions on Automatic Control, vol. 21, no. 1, pp. 25-34, 1976.

[3] B. A. Francis and W. M. Wonham, "The internal model principle of control theory," Automatica, vol. 12 , no. 5 , pp. $457-465,1976$.

[4] B. A. Francis, "The linear multivariable regulator problem," SIAM Journal on Control and Optimization, vol. 15 , no. 3, pp. 486-505, 1977.

[5] J. Huang, Nonlinear Output Regulation: Theory and Application, vol. 8 of Advances in Design and Control, SIAM, Philadelphia, Pa, USA, 2004.

[6] L. Qiu and E. J. Davison, "Performance limitations of nonminimum phase systems in the servomechanism problem," Automatica, vol. 29, no. 2, pp. 337-349, 1993.

[7] A. Saberi, A. A. Stoorvogel, and Z. Lin, "Generalized output regulation for linear systems," in Proceedings of the American Control Conference, pp. 3909-3914, IEEE, Alburquerque, NM, USA, 1997.

[8] W. M. Wonham, Linear Multivariable Control: A Geometric Approach, vol. 10 of Applications of Mathematics, Springer, New York, NY, USA, 2nd edition, 1979.

[9] Z. Wu and F. Ben Amara, "Parameterized regulator synthesis for bimodal linear systems based on bilinear matrix inequalities," Mathematical Problems in Engineering, vol. 2008, Article ID 341720, 22 pages, 2008.

[10] E. D. Sontag, "An introduction to the stabilization problem for parametrized families of linear systems," in Linear Algebra and Its Role in Systems Theory (Brunswick, Maine, 1984), vol. 47 of Contemporary Mathematics, pp. 369-400, American Mathematical Society, Providence, RI, USA, 1985.

[11] M. Hazewinkel, "(Fine) moduli (spaces) for linear systems: what are they and what are they good for?" in Geometrical Methods for the Study of Linear Systems, C. I. Byrnes and C. F. Martin, Eds., vol. 62, pp. 125-193, Reidel, Dordrecht, The Netherlands, 1980.

[12] C. I. Byrnes and C. F. Martin, Geometrical Methods for the Theory of Linear Systems, vol. 62 of NATO Science Series C, Springer, New York, NY, USA, 1980.

[13] C. I. Byrnes, "On the control of certain deterministic, infinite-dimensional systems by algebrogeometric techniques," American Journal of Mathematics, vol. 100, no. 6, pp. 1333-1381, 1978.

[14] E. D. Sontag, "On split realizations of response maps over rings," Information and Computation, vol. 37 , no. 1 , pp. 23-33, 1978.

[15] A. Tannenbaum, Invariance and System Theory: Algebraic and Geometric Aspects, vol. 845 of Lecture Notes in Mathematics, Springer, Berlin, Germany, 1981.

[16] M. L. J. Hautus and E. D. Sontag, "New results on pole-shifting for parametrized families of systems," Journal of Pure and Applied Algebra, vol. 40, no. 3, pp. 229-244, 1986.

[17] J. Ferrer and F. Puerta, "Global block-similarity and pole assignment of class $\mathbb{C}^{p}$," SIAM Journal on Matrix Analysis and Applications, vol. 19, no. 4, pp. 924-932, 1998.

[18] A. Compta, J. Ferrer, and M. Peña, "Local differentiable pole assignment," Linear and Multilinear Algebra, vol. 58, no. 5, pp. 563-569, 2010.

[19] J. Ferrer, Ma. I. García, and F. Puerta, “Differentiable families of subspaces," Linear Algebra and Its Applications, vol. 199, pp. 229-252, 1994. 


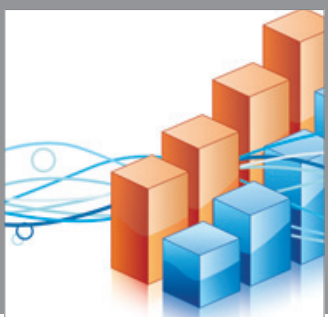

Advances in

Operations Research

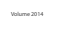

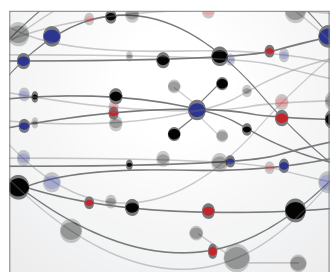

\section{The Scientific} World Journal
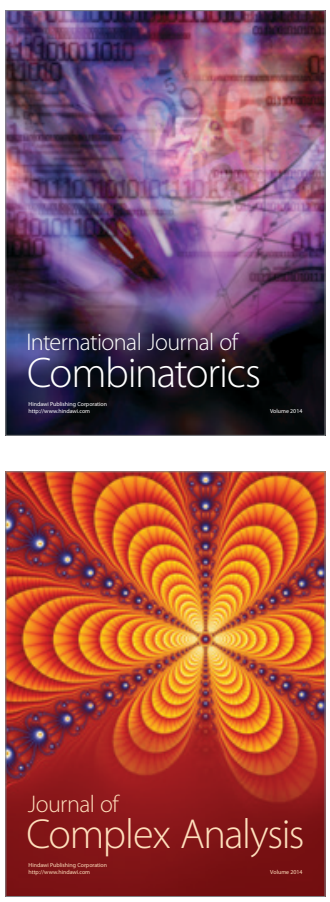

International Journal of

Mathematics and

Mathematical

Sciences
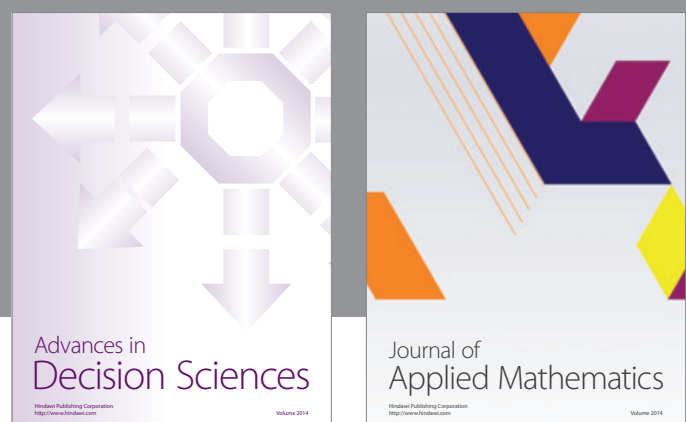

Journal of

Applied Mathematics
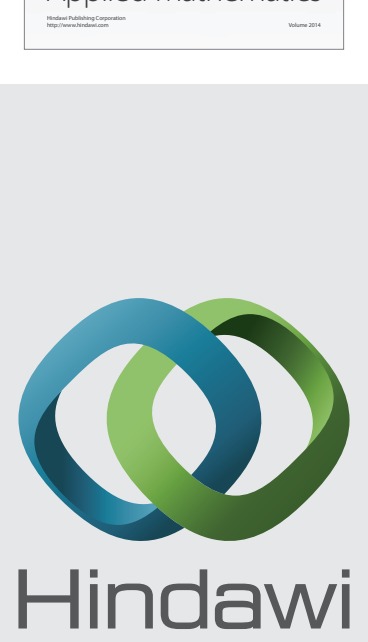

Submit your manuscripts at http://www.hindawi.com
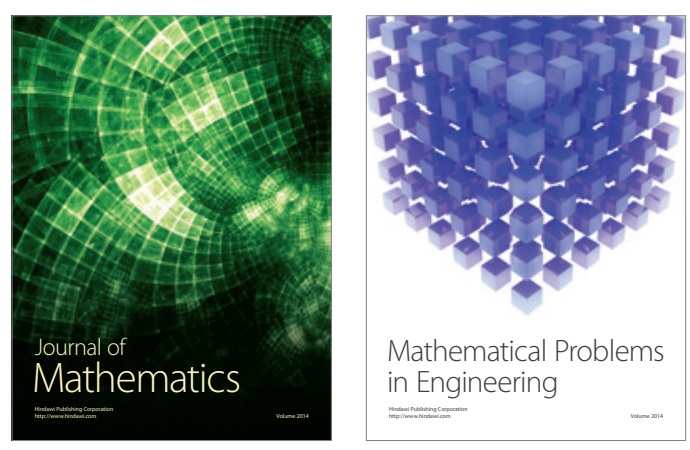

Mathematical Problems in Engineering
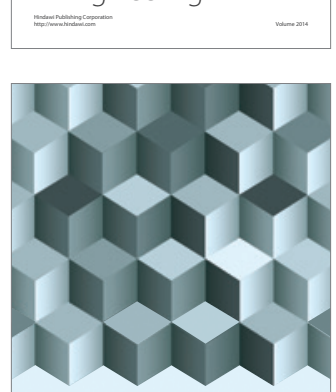

Journal of

Function Spaces
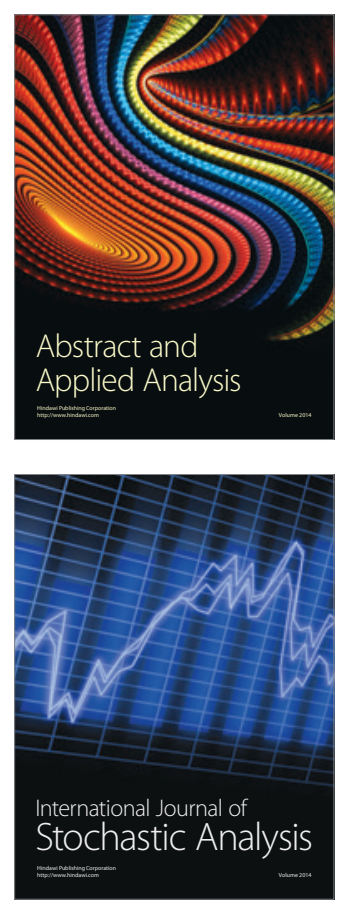

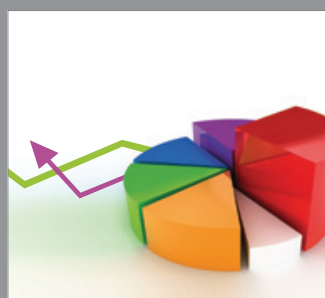

ournal of

Probability and Statistics

Promensencen
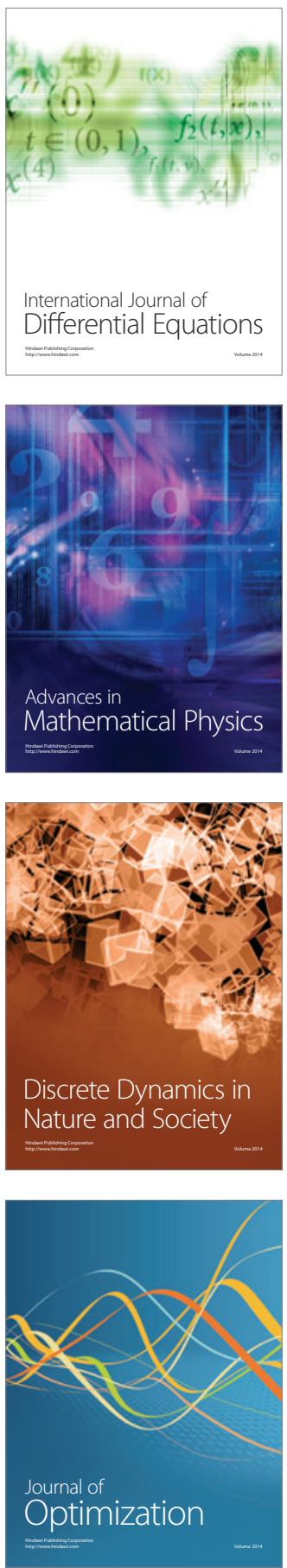